\title{
Gain in quantum cascade lasers and superlattices: A quantum transport theory
}

\author{
Andreas Wacker \\ Institut für Theoretische Physik, Technische Universität Berlin, Hardenbergstr. 36, 10623 Berlin, Germany
}

(Dated: 13. June 2002)

\begin{abstract}
Gain in current-driven semiconductor heterostructure devices is calculated within the theory of nonequilibrium Green functions. In order to treat the nonequilibrium distribution self-consistently the full two-time structure of the theory is employed without relying on any sort of Kadanoff-Baym Ansatz. The results are independent of the choice of the electromagnetic field if the variation of the self-energy is taken into account. Excellent quantitative agreement is obtained with the experimental gain spectrum of a quantum cascade laser. Calculations for semiconductor superlattices show that the simple 2-time miniband transport model gives reliable results for large miniband widths at room temperature.
\end{abstract}

PACS numbers: 05.60.Gg,42.55.Px,73.40.-c,78.67.-n

\section{INTRODUCTION}

The prospect of a semiconductor laser in the infrared and $\mathrm{THz}$ region has been one of the key reasons for the development and study of semiconductor heterostructure elements, since the first proposal of semiconductor superlattices in 1970 [1]. A possible gain mechanism may be based on two different ideas: (i) At certain electrical fields resonant tunneling between different subbands can lead to population inversion associated with gain at the transition energy 2]. This idea was realized in the quantum cascade laser [3], which has become an important device in the infrared region. Lasing in the $\mathrm{THz}$ region has been demonstrated very recently as well [4. For further details, see the review (5). (ii) The occurrence of negative differential conductivity in superlattices gives raise to gain in the low frequency range extending up to frequencies of the order of the Bloch frequency [6]. Despite a strong effort by several groups all over the world, this idea is still not realized. The difficulty in its realization is attributed to the instability of the operating state leading to domain formation [7], which may be circumvented by different strategies [8, 9, 10]. For further references and a detailed discussion see Ref. 11 .

These concepts for gain are commonly described in different ways: (i) An external electromagnetic field causes transitions between different levels, where the transition rate $R$ is evaluated by Fermi's golden rule. If stimulated emission dominates stimulated absorption (i.e., for population inversion), gain occurs at the transition frequency. For a pair of states $\alpha, \beta$ with energies $E_{\alpha}, E_{\beta}$, occupation probabilities $f_{\alpha}, f_{\beta}$, and a dipole matrix element $z_{\alpha \beta}$, the contribution to the material gain (i.e., increase of light

*Electronic address: wacker@physik.tu-berlin.de intensity per length) is given by

$$
\begin{aligned}
G_{m}(\omega) & =\frac{1}{V} \frac{R_{\alpha \rightarrow \dot{\beta}}^{\text {stim. em. }}-R_{\beta \rightarrow \alpha}^{\text {stim. abs. }}}{\text { Photon flux per area }} \\
& =\frac{\pi \omega\left|e z_{\alpha \beta}\right|^{2}}{\sqrt{\epsilon_{r}} c \epsilon_{0} V} \delta\left(E_{\alpha}-E_{\beta}-\hbar \omega\right)\left(f_{\alpha}-f_{\beta}\right),
\end{aligned}
$$

where $V$ is the normalization volume, $e<0$ is the electron charge, $c$ is the vacuum speed of light, and $\epsilon_{r}$ the background dielectric constant.

(ii) Transport theory in the presence of alternating electric fields provides the complex dynamical conductance $\sigma(\omega)$. Standard electrodynamics (see, e.g., section 7.5 of Ref. [12]) gives the material gain (the negative absorption coefficient $\alpha$, which is assumed to be small compared to the wave vector here)

$$
G_{m}(\omega) \approx-\frac{\Re\{\sigma(\omega)\}}{c \epsilon_{0} \sqrt{\epsilon_{r}-\Im\{\sigma(\omega)\} / \epsilon_{0} \omega}} .
$$

Thus, gain is equivalent to a negative differential conductivity in the respective frequency range. This concept has been frequently applied to superlattice transport. (For superlattices Eq. (17) gives zero gain in the basis of Wannier Stark states, as the translational symmetry gives identical $f_{\alpha}$ for all Wannier-Stark levels.) Here the current is either evaluated from a semiclassical miniband transport model [6, 7, 9, 10] or by sequential tunneling between different layers [8, 13, 14. While these approaches use a macroscopic current density in Eq. (2), the consideration of polarization currents allows for a derivation of Eq. (11), see, e.g., Ref. 15.

For complicated semiconductor heterostructures, with a variety of different tunneling and optical transitions, gain may result both from optical transitions in the spirit of (i) and macroscopic currents in the spirit of (ii). The aim of this paper is to show the feasibility of a general approach based on a quantum transport theory which treats both mechanisms on a equal footing.

The method of nonequilibrium Green functions 16] is used here, which has been frequently applied to light emission in semiconductors (see, e.g., Refs. 17, 18, 19, 20 
and references given therein). While these works consider transitions between the conduction and valence band, the focus in this paper is on transitions within the conduction band in current-driven heterostructure devices. The calculations are performed in two steps: First the transport problem is solved by evaluating a self-consistent stationary solution of the quantum kinetic equations for a given applied bias. This provides us with the Green functions describing the electronic state far from equilibrium. In a second step, an additional weak radiation field is taken into account. The time-dependent kinetic equations are linearized around the stationary nonequilibrium state in oder to study the linear response of the current-driven system. The formulation used here employs the full twotime structure of the theory without any sort of (generalized) Kadanoff-Baym ansatz [21. The capability of the approach is demonstrated by calculations of the gain spectra in a quantum cascade laser and a superlattice.

\section{THEORY}

We describe a general system by a set of orthonormal states labeled $\alpha$ with energies $E_{\alpha}$ and write the Hamiltonian in the form:

$$
\hat{H}=\sum_{\alpha} E_{\alpha} \hat{a}_{\alpha}^{\dagger} \hat{a}_{\alpha}+\sum_{\alpha \beta} U_{\alpha \beta}(t) \hat{a}_{\alpha}^{\dagger} \hat{a}_{\beta}+\hat{H}_{\text {scatt }}
$$

where $\hat{a}_{\alpha}$ and $\hat{a}_{\alpha}^{\dagger}$ are electron annihilation and creation operators in the state $\alpha$. $U_{\alpha \beta}(t)$ represents the matrix elements of the kinetic energy and the potential part of the Hamiltonian $\hat{H}$. In the following, $U(t)$ will be split into a constant part $\tilde{U}$ and a small time-dependent perturbation $\delta U(t)$ describing the interaction with a radiation field. Finally, $\hat{H}_{\text {scatt }}$ contains the part of the Hamiltonian which will be solved perturbatively (such as impurity, phonon, or electron-electron scattering matrix elements).

Within the theory of nonequilibrium Green functions 16, 18 the key quantities are the correlation function (or 'lesser' Green function)

$$
G_{\alpha_{1}, \alpha_{2}}^{<}\left(t_{1}, t_{2}\right)=\mathrm{i}\left\langle\hat{a}_{\alpha_{2}}^{\dagger}\left(t_{2}\right) \hat{a}_{\alpha_{1}}\left(t_{1}\right)\right\rangle
$$

and the retarded/advanced Green functions

$$
\begin{aligned}
G_{\alpha_{1}, \alpha_{2}}^{\mathrm{ret} / \mathrm{adv}} & \left(t_{1}, t_{2}\right)=\mp \mathrm{i} \Theta\left[ \pm\left(t_{1}-t_{2}\right)\right] \\
& \times\left\langle\hat{a}_{\alpha_{1}}\left(t_{1}\right) \hat{a}_{\alpha_{2}}^{\dagger}\left(t_{2}\right)+\hat{a}_{\alpha_{2}}^{\dagger}\left(t_{2}\right) \hat{a}_{\alpha_{1}}\left(t_{1}\right)\right\rangle,
\end{aligned}
$$

respectively, where the Heisenberg picture is used.

First consider a stationary state with a time independent matrix $U(t)=\tilde{U}$, neglecting the radiation field. In this case all functions depend only on the time difference $t_{1}-t_{2}$ [these stationary state functions are labeled by a tilde, e.g., $\tilde{G}\left(t_{1}-t_{2}\right)$, in the following], and it is convenient to work in Fourier space defined by

$$
\tilde{G}_{\alpha_{1}, \alpha_{2}}(E)=\frac{1}{\hbar} \int \mathrm{d} t \mathrm{e}^{\mathrm{i} E t / \hbar} \tilde{G}_{\alpha_{1}, \alpha_{2}}(t) .
$$

Then Eqs. A3, A4 of the appendix become the matrix equations

$$
\begin{array}{r}
\left(E-E_{\alpha_{1}}\right) \tilde{G}_{\alpha_{1}, \alpha_{2}}^{\mathrm{ret}}(E)-\sum_{\beta} \tilde{U}_{\alpha_{1}, \beta} \tilde{G}_{\beta, \alpha_{2}}^{\mathrm{ret}}(E) \\
=\delta_{\alpha_{1}, \alpha_{2}}+\sum_{\beta} \tilde{\Sigma}_{\alpha_{1}, \beta}^{\mathrm{ret}}(E) \tilde{G}_{\beta, \alpha_{2}}^{\mathrm{ret}}(E) \\
\tilde{G}_{\alpha_{1}, \alpha_{2}}^{<}(E)=\sum_{\beta, \beta^{\prime}} \tilde{G}_{\alpha_{1}, \beta}^{\mathrm{ret}}(E) \tilde{\Sigma}_{\beta, \beta^{\prime}}^{<}(E) \tilde{G}_{\beta^{\prime}, \alpha_{2}}^{\mathrm{adv}}(E) .
\end{array}
$$

These have to be solved self-consistently together with the equations for the self-energies which are functionals of the Green functions

$$
\tilde{\Sigma}(E)=\mathcal{F}_{E}\left\{\tilde{G}^{\mathrm{ret}}\left(E^{\prime}\right), \tilde{G}^{<}\left(E^{\prime}\right)\right\} .
$$

This standard approach allows for a self-consistent evaluation of the Green functions $\tilde{G}$ in a nonequilibrium situation caused by an applied bias (contained in $\tilde{U}$ ). Details, such as the specific form of the functionals in the self-consistent Born approximation, can be found in, e.g., 11, 22, 23.

The current density (in the $z$-direction) can be evaluated from the expectation value of the momentum operator divided by the mass:

$$
\begin{aligned}
J_{z} & =\frac{e}{V}\left\langle\frac{\hat{p}_{z}}{m}\right\rangle=\frac{e}{V} \frac{\mathrm{i}}{\hbar}\langle[\hat{H}, \hat{z}]\rangle \\
& =\frac{e}{\hbar V} \sum_{\alpha \beta} W_{\alpha \beta} G_{\beta \alpha}^{<}(t, t)+\text { Scattering currents }
\end{aligned}
$$

where $W_{\alpha \beta}=\sum_{\gamma}\left(U_{\alpha \gamma} z_{\gamma \beta}-z_{\alpha \gamma} U_{\gamma \beta}\right)$. The scattering currents result from the scattering part of the Hamiltonian and can be expressed in terms of self-energies. Details will be given elsewhere.

Now we consider the influence of an additional timedependent potential

$$
\delta \mathbf{U}(t)=\int \frac{\mathrm{d} \omega}{2 \pi} \delta \mathbf{U}(\omega) \mathrm{e}^{-\mathrm{i} \omega t}
$$

in the Hamiltonian. (Bold capital symbols denote matrices in the state indices $\alpha, \beta)$. We treat the change of the system in linear response, and set

$$
G\left(t_{1}, t_{2}\right)=\tilde{G}\left(t_{1}-t_{2}\right)+\delta G\left(t_{1}, t_{2}\right)
$$

The chance in the self-energies is described within the linearization of the functional (here in the time domain)

$$
\Sigma\left(t_{1}, t_{2}\right)=\mathcal{F}_{t}\{\tilde{G}+\delta G\} \approx \tilde{\Sigma}\left(t_{1}-t_{2}\right)+\delta \Sigma\left(t_{1}, t_{2}\right) .
$$

Such a decomposition has been used in Ref. 17 for Hartree-Fock self-energies, e.g.. In this case, the timedependence of the self-energies allows for a reduction to density matrix equations by setting $t_{1}=t_{2}$. In contrast, in our case, where scattering effects are considered, $\delta G$ 
exhibits an explicit time dependence in both arguments. This two time structure is fully taken into account here. Therefore we apply the Fourier decomposition in both times via

$$
\delta \mathbf{G}\left(t_{1}, t_{2}\right)=\int \frac{\mathrm{d} \omega}{2 \pi} \mathrm{e}^{-\mathrm{i} \omega t_{1}} \int \frac{\mathrm{d} E}{2 \pi} \delta \mathbf{G}(\omega, E) \mathrm{e}^{-\mathrm{i} E\left(t_{1}-t_{2}\right) / \hbar}
$$

The same decomposition is used for $\delta \Sigma$. It is shown in appendix $\mathrm{C}$ that $\delta G$ is determined within linear response by the following equations:

$$
\begin{aligned}
\delta \mathbf{G}^{\mathrm{ret} / \operatorname{adv}}(\omega, E) & =\tilde{\mathbf{G}}^{\mathrm{ret} / \operatorname{adv}}(E+\hbar \omega)\left[\delta \mathbf{U}(\omega)+\delta \boldsymbol{\Sigma}^{\mathrm{ret} / \mathrm{adv}}(\omega, E)\right] \tilde{\mathbf{G}}^{\mathrm{ret} / \mathrm{adv}}(E) \\
\delta \mathbf{G}^{<}(\omega, E) & =\tilde{\mathbf{G}}^{\mathrm{ret}}(E+\hbar \omega) \delta \mathbf{U}(\omega) \tilde{\mathbf{G}}^{<}(E)+\tilde{\mathbf{G}}^{<}(E+\hbar \omega) \delta \mathbf{U}(\omega) \tilde{\mathbf{G}}^{\mathrm{adv}}(E) \\
& +\tilde{\mathbf{G}}^{\mathrm{ret}}(E+\hbar \omega) \delta \boldsymbol{\Sigma}^{\mathrm{ret}}(\omega, E) \tilde{\mathbf{G}}^{<}(E)+\tilde{\mathbf{G}}^{\mathrm{ret}}(E+\hbar \omega) \delta \boldsymbol{\Sigma}^{<}(\omega, E) \tilde{\mathbf{G}}^{\mathrm{adv}}(E) \\
& +\tilde{\mathbf{G}}^{<}(E+\hbar \omega) \delta \boldsymbol{\Sigma}^{\mathrm{adv}}(\omega, E) \tilde{\mathbf{G}}^{\mathrm{adv}}(E)
\end{aligned}
$$

The changes in self-energy $\delta \Sigma$ are functionals of $\delta G$, which have to be evaluated self-consistently with $\delta G$. The derivation of the functionals from the linearization of Eq. (13) is straightforward and can be performed for arbitrary self-energies. The situation is particularly simple for the self-consistent Born approximation, where the functional $\mathcal{F}$ is linear in $G$, and one finds: $\delta \Sigma(\omega, E)=$ $\mathcal{F}_{E}\left\{\delta G^{\text {ret }}\left(\omega, E^{\prime}\right), \delta G^{<}\left(\omega, E^{\prime}\right)\right\}$ with the same functional as Eq. (9). (Note, that $\delta \mathbf{G}^{\text {adv }}\left(\omega, E^{\prime}\right)=\left[\delta \mathbf{G}^{\text {ret }}\left(\omega, E^{\prime}\right)\right]^{\dagger}$ does not hold for the time-dependent quantities. Thus, the advanced quantities should be calculated explicitely)

The terms related to $\delta \boldsymbol{\Sigma}$ correspond to the ladder corrections in the evaluation of diagrams for the Kubo formula in equilibrium. It is a general advantage of linear response within nonequilibrium Green functions that these terms are obtained directly, see also [18, 24].

Now we consider the linear response to an external radiation field where the electric field points in the $z$ direction. This gives additional terms in the Hamiltonian $\hat{H}=(\hat{\vec{p}}-e \vec{A})^{2} / 2 m+V(\vec{r})+e \varphi(\vec{r})$, where $\vec{A}, \varphi$ are the electromagnetic potentials. Neglecting quadratic terms in the radiation field and terms containing $k$ (i.e., assuming that the wavelength is large compared to the size of the active region), the following perturbation potentials are obtained (see Appendix D): In the Coulomb gauge

$$
\delta U_{\alpha, \beta}(\omega)=\frac{e}{\mathrm{i} \omega} F(\omega)\left(\frac{\hat{p}_{z}}{m}\right)_{\alpha, \beta} \approx-\frac{e F(\omega)}{\hbar \omega} W_{\alpha, \beta}
$$

neglecting the scattering part of $\hat{H}$; in the Lorentz gauge

$$
\delta U_{\alpha, \beta}(\omega)=-e F(\omega) z_{\alpha, \beta}
$$

Finally, the change in current density is given by the change in Eq. (10)

$$
\begin{aligned}
& \delta J_{z}(\omega)=\frac{e}{\hbar V} \int \frac{\mathrm{d} E}{2 \pi} \operatorname{Tr}\{ {[\delta \mathbf{U}(\omega) \cdot \mathbf{Z}-\mathbf{Z} \cdot \delta \mathbf{U}(\omega)] } \\
&\left.\cdot \tilde{\mathbf{G}}^{<}(E)+\mathbf{W} \cdot \delta \mathbf{G}^{<}(\omega, E)\right\} .
\end{aligned}
$$

The scattering current in Eq. (10) has not been included here. With these ingredients, one obtains the complex conductivity $\sigma(\omega)=\delta J(\omega) / F(\omega)$, as well as the gain coefficient via Eq. (2).

\section{RESULTS}

In the following we consider two different structures: (i) The quantum-cascade-laser structure of Ref. 25. (ii) The superlattice used in Ref. 10 with $2 \mathrm{~nm} \mathrm{Al}_{0.3} \mathrm{Ga}_{0.7} \mathrm{As}$ barriers and $8 \mathrm{~nm}$ GaAs wells and a doping of $n=$ $10^{16} / \mathrm{cm}^{3}$. In both cases, we use a basis set consisting of products of Wannier functions (in the growth direction $z$ ) and plane waves with wave vector $\mathbf{k}$ (in the $(x, y)$ plane), and obtain $U_{\alpha \beta}$ from nominal sample parameters [11]. The self-consistent solution for the electrostatic potential is included as well.

For the scattering Hamiltonian we include interfaceroughness scattering and phonon scattering (including both optical phonons and a second phonon with low energy to mimic acoustic phonons). The self-energies are evaluated in the self-consistent Born approximation. Furthermore, we use momentum-independent scattering matrix elements (evaluated for a typical momentum transfer) and diagonal self-energies. In this approximation the self-energies are not k-dependent, which significantly reduces the numerical effort. Results and further details for superlattices [11, 26] and quantum cascade lasers 27] have been given previously. The current-field characteristics are displayed in Fig. 1 $(a, b)$ for both structures, which 

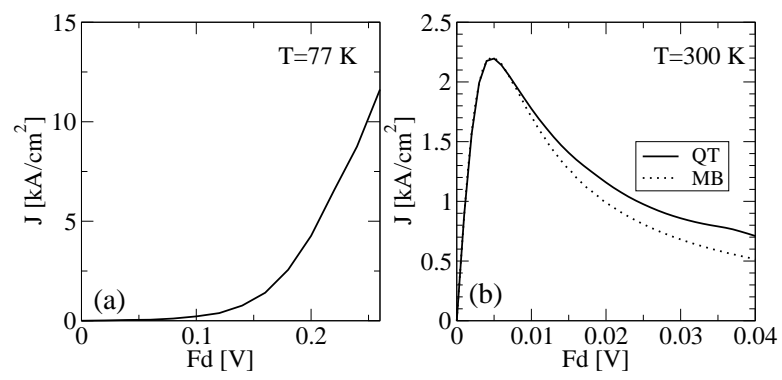

FIG. 1: Current density versus potential drop per period for the quantum cascade laser of Ref. 25 (a) and the superlattice structure of Ref. 10 (b). The full line gives the result of the quantum transport model (QT), while the dashed line is evaluated with the simple 2-time miniband model (MB), Eq. 20.).

agree reasonably well with the respective experimental data. Note that domain formation in the region of negative differential conductivity modifies the behavior of the experimental curve for the superlattice. Such effects are beyond the scope of the general approach discussed here, but can be easily studied using simpler transport models [11, 28, 29]. Similar results for the current-field relation of this quantum cascade laser have been reported in Ref. 30.

For the superlattice structure, the simple 2-time miniband transport model [6, 31 gives

$$
J_{\mathrm{M} B}(F)=e n \frac{d \Delta}{2 \hbar} \frac{I_{1}\left(\Delta / 2 k_{B} T\right)}{I_{0}\left(\Delta / 2 k_{B} T\right)} \frac{e F d \hbar / \tau_{e}}{(e F d)^{2}+\hbar^{2} / \tau_{e} \tau_{m}}
$$

for a non-degenerate electron gas. Here $F$ is the electrical field, $\Delta$ the miniband width and $d$ the period of the superlattice. $\tau_{e}=0.17 \mathrm{ps}$ and $\tau_{m}=0.113 \mathrm{ps}$ represent fitted, phenomenological energy and momentum scattering times. $I_{j}(x)$ are the modified Bessel functions. In Fig. 1. (b) this model shows reasonable agreement with the full quantum transport calculation for $|e F d| \lesssim \Delta / 2=11$ $\mathrm{meV}$, the field range where miniband transport holds true [32]. (For lower temperatures the shape of the currentfield relation becomes more complicated both in the semiclassical Boltzmann and the quantum transport model [26] so that the simple 2-time model cannot fit the data.)

In the following the response to an external radiation field is studied: First let us use the Coulomb gauge and neglect the terms with $\delta \Sigma$ in Eq. (16). The gain spectrum for the quantum cascade laser of Ref. 25 is displayed in Fig. 2. Gain sets in for current densities above 0.8 $\mathrm{kA} / \mathrm{cm}^{2}$ and increases with current. The peak gain coefficient is $57 \mathrm{~cm}^{-1}$ at $6.5 \mathrm{kA} / \mathrm{cm}^{2}$ and a photon energy of $130 \mathrm{meV}$, in excellent agreement with the findings of Ref. 25 (estimated losses divided by confinement factor $63 \mathrm{~cm}^{-1}$ and threshold current $J_{\mathrm{th}}=7.2 \mathrm{kA} / \mathrm{cm}^{2}$, lasing at $131 \mathrm{meV}$ ). The width of the gain spectrum agrees well with the findings of Ref. 33 .

Results for the superlattice are given in Fig. 3. The real part of the conductivity essentially follows the result

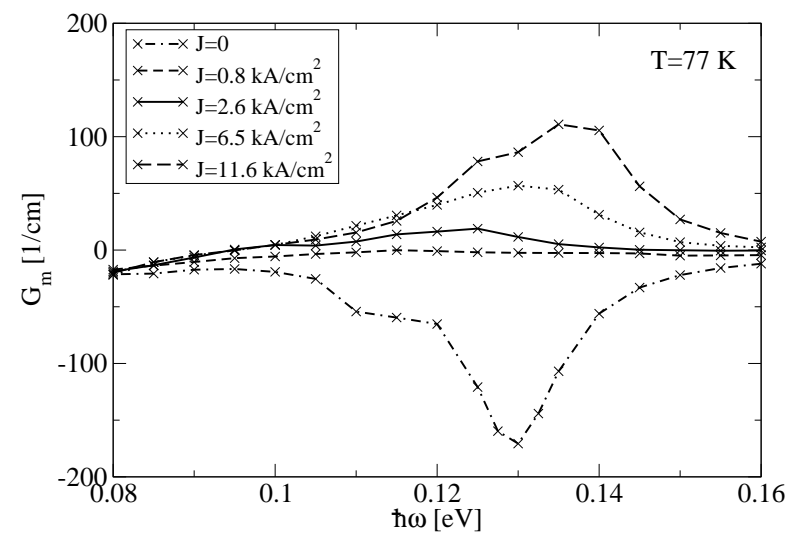

FIG. 2: Gain spectrum for the quantum cascade laser of Ref. 25 evaluated from the quantum transport model with Coulomb gauge and neglecting self-energy corrections.

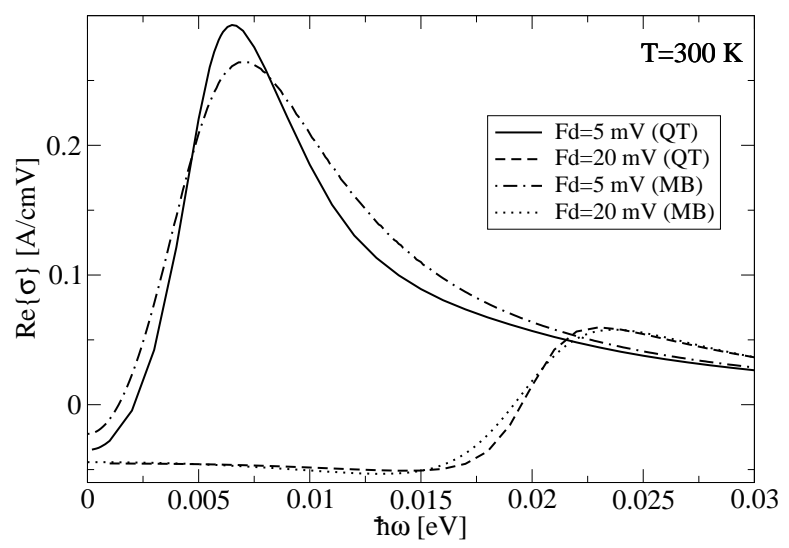

FIG. 3: Real part of the dynamical conductance for the superlattice structure of Ref. 10. Full and dashed line: Result from the quantum transport model with Coulomb gauge and neglecting self-energy corrections for $F d=5 \mathrm{mV}$ and $20 \mathrm{mV}$, respectively. Dotted and dash-dotted line: Corresponding results from the 2-time miniband model [6].

from the simple 2-time miniband model [6]

$$
\sigma(\omega)=\frac{J_{\mathrm{MB}}(F)}{F} \frac{1-\omega_{B} \tau_{m} \tau_{e}-\mathrm{i} \omega \tau_{e}}{\left(\omega_{B}^{2}-\omega^{2}\right) \tau_{m} \tau_{e}+1-\mathrm{i} \omega\left(\tau_{m}+\tau_{e}\right)}
$$

with $\omega_{B}=e F d / \hbar$. Thus, this simple model gives, at least for wide minibands and high temperatures, reliable results.

Now we want to study the relevance of the terms $\delta \Sigma$ in Eq. (16), which have been neglected so far. Fig. 1 shows that their inclusion barely changes the result in the Coulomb gauge (the full and dashed lines are almost indistinguishable). Therefore these self-energy corrections may be neglected (at least for diagonal self-energies assumed here) allowing for a significant reduction of the numerical effort. In contrast, in the case of the Lorentz gauge [34, the terms evaluated with self-energy corrections (dash-dotted line) differ substantially from the simpler version without these terms (dotted line). The differ- 

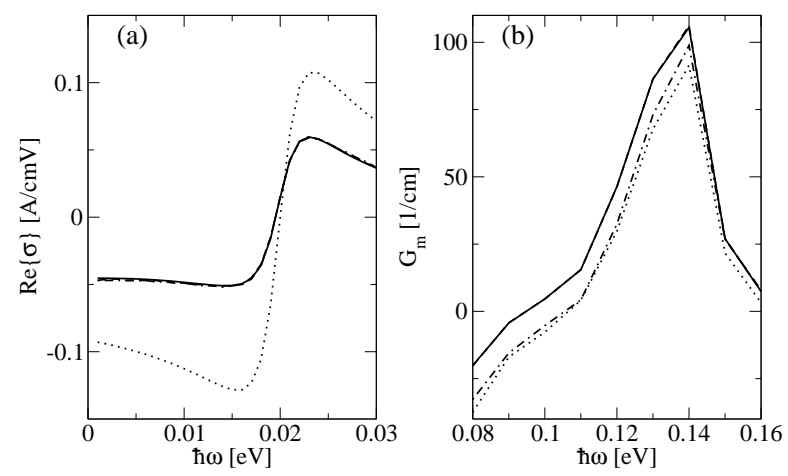

FIG. 4: Comparison of different choices of the gauge and selfenergy corrections $\delta \Sigma$ for (a) the superlattice at $F d=20$ and (b) the quantum cascade laser at $F d=260 \mathrm{mV}$. Full line: Coulomb gauge neglecting $\delta \Sigma$; Dotted line: Lorentz gauge neglecting $\delta \Sigma$; Dashed line: Coulomb gauge with $\delta \Sigma$ (falls together with the full line); Dash-dotted line: Lorentz gauge with $\delta \Sigma$.

ence is particularly strong in the case of the superlattice, where the diagonal terms in the perturbation potential (18) are essential for the transition. Furthermore, the results of Coulomb gauge and Lorentz gauge are almost identical if the self-energy corrections are included. This demonstrates that the inclusion of these terms is essential to obtain a consistent theory, which must be independent of the choice of the gauge.

\section{CONCLUSION}

A formulation of the theory of nonequilibrium Green functions has been given which allows for a quantitative description of current-induced gain in semiconductor heterostructures. Quantitative agreement with the experiment for the gain spectrum of a quantum cascade laser was obtained. The standard 2-time miniband model for the superlattice could be verified for a large miniband width and under room temperature operation. The numerical results are not sensitive to the choice of the gauge if the variations of self-energy terms are taken into account. These terms are of particular importance for the Lorentz gauge, while their contribution is small in the Coulomb gauge in the calculations presented here.

\section{Acknowledgments}

Helpful discussions with A. Knorr, A.-P. Jauho, S.C. Lee, M. Pereira and E. Schöll as well as financial support by DFG within FOR394 are gratefully acknowledged.

\section{APPENDIX A: DERIVATION OF THE EQUATIONS FOR THE STATIONARY STATE}

The Green functions are determined by the Dyson Equation (see, e.g., chapter 5 of Ref. 18)

$$
\mathrm{i} \hbar \frac{\partial}{\partial t_{1}} G_{\alpha_{1}, \alpha_{2}}^{\mathrm{ret} / \mathrm{adv}}\left(t_{1}, t_{2}\right)-\sum_{\beta} U_{\alpha_{1}, \beta}\left(t_{1}\right) G_{\beta, \alpha_{2}}^{\mathrm{ret} / \mathrm{adv}}\left(t_{1}, t_{2}\right)-\sum_{\beta} \int \frac{\mathrm{d} t}{\hbar} \Sigma_{\alpha_{1}, \beta}^{\mathrm{ret} / \mathrm{adv}}\left(t_{1}, t\right) G_{\beta, \alpha_{2}}^{\mathrm{ret} / \mathrm{adv}}\left(t, t_{2}\right)
$$

$$
=\hbar \delta\left(t_{1}-t_{2}\right) \delta_{\alpha_{1}, \alpha_{2}}
$$

and the Keldysh relation, see also Appendix B.

$$
G_{\alpha_{1}, \alpha_{2}}^{<}\left(t_{1}, t_{2}\right)=\sum_{\beta, \beta^{\prime}} \int \frac{\mathrm{d} t}{\hbar} \int \frac{\mathrm{d} t^{\prime}}{\hbar} G_{\alpha_{1}, \beta}^{\mathrm{ret}}\left(t_{1}, t\right) \Sigma_{\beta, \beta^{\prime}}^{<}\left(t, t^{\prime}\right) G_{\beta^{\prime}, \alpha_{2}}^{\mathrm{adv}}\left(t^{\prime}, t_{2}\right)
$$

Now we consider the stationary state, where $G\left(t_{1}, t_{2}\right)=\tilde{G}\left(t_{1}-t_{2}\right)$. Eqs. A1 A2 become:

$$
\begin{gathered}
\mathrm{i} \hbar \frac{\partial}{\partial t} \tilde{G}_{\alpha_{1}, \alpha_{2}}^{\mathrm{ret} / \mathrm{adv}}(t)-\sum_{\beta} \tilde{U}_{\alpha_{1}, \beta} \tilde{G}_{\beta, \alpha_{2}}^{\mathrm{ret} / \mathrm{adv}}(t)-\sum_{\beta} \int \frac{\mathrm{d} t^{\prime}}{\hbar} \tilde{\Sigma}_{\alpha_{1}, \beta}^{\mathrm{ret} / \mathrm{adv}}\left(t-t^{\prime}\right) \tilde{G}_{\beta, \alpha_{2}}^{\mathrm{ret} / \mathrm{adv}}\left(t^{\prime}\right)=\hbar \delta(t) \delta_{\alpha_{1}, \alpha_{2}} \\
\tilde{G}_{\alpha_{1}, \alpha_{2}}^{<}(t)=\sum_{\beta, \beta^{\prime}} \int \frac{\mathrm{d} t^{\prime}}{\hbar} \int \frac{\mathrm{d} t^{\prime \prime}}{\hbar} \tilde{G}_{\alpha_{1}, \beta}^{\mathrm{ret}}\left(t-t^{\prime}\right) \tilde{\Sigma}_{\beta, \beta^{\prime}}^{<}\left(t^{\prime}-t^{\prime \prime}\right) \tilde{G}_{\beta^{\prime}, \alpha_{2}}^{\mathrm{adv}}\left(t^{\prime \prime}\right) .
\end{gathered}
$$

and their Fourier-transformation (6) provides us with Eqs. (77,8).

\section{APPENDIX B: A REMARK ON EQ. (5.11) OF REF. 18}

Eq. (A2) is a particular solution of the inhomogeneous differential equations (5.3) of Ref. 18, which are given more explicitly in Eqs. (106-107) of Ref. 11 in the notation used here. The Keldysh relation (5.11) derived in Ref. 18 
contains an additional term:

$$
\begin{aligned}
& \mathbf{G}_{\text {add }}^{<}\left(t_{1}, t_{2}\right)=\int \frac{\mathrm{d} t_{b}}{\hbar} \int \frac{\mathrm{d} t_{c}}{\hbar}[\left.\hbar \delta\left(t_{1}-t_{b}\right) \mathbf{1}+\mathbf{G}^{\mathrm{ret}}\left(t_{1}, t_{b}\right) \mathbf{U}\left(t_{b}\right)+\int \frac{\mathrm{d} t_{a}}{\hbar} \mathbf{G}^{\mathrm{ret}}\left(t_{1}, t_{a}\right) \boldsymbol{\Sigma}^{\mathrm{ret}}\left(t_{a}, t_{b}\right)\right] \\
& \times \mathbf{G}_{0}^{<}\left(t_{b}, t_{c}\right)\left[\hbar \delta\left(t_{c}-t_{2}\right) \mathbf{1}+\mathbf{U}\left(t_{c}\right) \mathbf{G}^{\mathrm{adv}}\left(t_{c}, t_{2}\right)+\int \frac{\mathrm{d} t_{d}}{\hbar} \boldsymbol{\Sigma}^{\mathrm{ret}}\left(t_{c}, t_{d}\right) \mathbf{G}^{\mathrm{adv}}\left(t_{d}, t_{2}\right)\right]
\end{aligned}
$$

This term constitutes a homogeneous solution of the inhomogeneous differential equations (for fixed $\Sigma$ and $G^{\text {ret/adv }}$ ) which guarantees that $G^{<} \rightarrow G_{0}^{<}$for $t_{1}, t_{2} \rightarrow-\infty$. This satisfies a general assumption underlying the perturbation expansion of the $S$-matrix. In the following we show that $G_{\text {add }}^{<}$vanishes for finite times $t_{1}, t_{2}$.

Using Eqs. $(108,109)$ of Ref. 11 one obtains

$$
\begin{aligned}
\mathbf{G}_{\text {add }}^{<}\left(t_{1}, t_{2}\right)= & \int \frac{\mathrm{d} t_{a}}{\hbar} \int \frac{\mathrm{d} t_{c}}{\hbar} \mathbf{G}^{\mathrm{ret}}\left(t_{1}, t_{a}\right)\left(-\mathrm{i} \hbar \frac{\partial^{\mathrm{left}}}{\partial t_{a}}-\mathbf{E}\right) \mathbf{G}_{0}^{<}\left(t_{a}, t_{c}\right)\left(\mathrm{i} \hbar \frac{\partial}{\partial t_{c}}-\mathbf{E}\right) \mathbf{G}^{\mathrm{adv}}\left(t_{c}, t_{2}\right) \\
= & \int \frac{\mathrm{d} t_{a}}{\hbar} \int \frac{\mathrm{d} t_{c}}{\hbar} \mathbf{G}^{\mathrm{ret}}\left(t_{1}, t_{a}\right)\left(\mathrm{i} \hbar \frac{\partial}{\partial t_{a}}-\mathbf{E}\right) \mathbf{G}_{0}^{<}\left(t_{a}, t_{c}\right)\left(\mathrm{i} \hbar \frac{\partial}{\partial t_{c}}-\mathbf{E}\right) \mathbf{G}^{\text {adv }}\left(t_{c}, t_{2}\right) \\
& -\mathrm{i}\left[\int \frac{\mathrm{d} t_{c}}{\hbar} \mathbf{G}^{\mathrm{ret}}\left(t_{1}, t_{a}\right) \mathbf{G}_{0}^{<}\left(t_{a}, t_{c}\right)\left(\mathrm{i} \hbar \frac{\partial}{\partial t_{c}}-\mathbf{E}\right) \mathbf{G}^{\text {adv }}\left(t_{c}, t_{2}\right)\right]_{t_{a}=-\infty}^{t_{a}=\infty},
\end{aligned}
$$

where $\partial^{\text {left }}$ means that the derivative operates to the left. In the second line, partial integration has been used. As $\left(\mathrm{i} \hbar \frac{\partial}{\partial t_{a}}-\mathbf{E}\right) \mathbf{G}_{0}^{<}\left(t_{a}, t_{c}\right)=0$, the first term vanishes. Furthermore, at the upper bound $\mathbf{G}^{\text {ret }}\left(t_{1}, \infty\right)=0$ holds for finite times $t_{1}$. Applying the same manipulations for $t_{c}$, we find

$$
\begin{aligned}
\mathbf{G}_{\text {add }}^{<}\left(t_{1}, t_{2}\right) & =\mathrm{i} \int \frac{\mathrm{d} t_{c}}{\hbar} \mathbf{G}^{\text {ret }}\left(t_{1},-\infty\right) \mathbf{G}_{0}^{<}\left(-\infty, t_{c}\right)\left(\mathrm{i} \hbar \frac{\partial}{\partial t_{c}}-\mathbf{E}\right) \mathbf{G}^{\text {adv }}\left(t_{c}, t_{2}\right) \\
& =\mathbf{G}^{\text {ret }}\left(t_{1},-\infty\right) \mathbf{G}_{0}^{<}(-\infty,-\infty) \mathbf{G}^{\text {adv }}\left(-\infty, t_{2}\right)
\end{aligned}
$$

For a real system the retarded and advanced Green functions decay for large time differences and we find $\mathbf{G}_{\text {add }}^{<}\left(t_{1}, t_{2}\right) \rightarrow$ 0 for finite $t_{1}, t_{2}$. Therefore this additional term is spurious.

A second, more physical argument relates to the idea, that the stationary state of a real physical system should not depend on the initial conditions used in a formal theory. Except for specific situations exhibiting a hysteresis, scattering processes will install a stationary state which is independent of initial conditions. Therefore all additional homogeneous solutions should reflect transient effects, which vanish if the initial state is chosen at $t_{1}=-\infty$ and $t_{2}=-\infty$

\section{APPENDIX C: DERIVATION OF EQS. (15, 16,}

Setting $U(t)=\tilde{U}+\delta U(t), G\left(t_{1}, t_{2}\right)=\tilde{G}\left(t_{1}-t_{2}\right)+\delta G\left(t_{1}, t_{2}\right)$ and $\Sigma\left(t_{1}, t_{2}\right)=\tilde{\Sigma}\left(t_{1}-t_{2}\right)+\delta \Sigma\left(t_{1}, t_{2}\right)$ in Eqs. (A1, A2) gives in linear order $\delta U$ :

$$
\begin{aligned}
\mathrm{i} \hbar \frac{\partial}{\partial t_{1}} \delta G_{\alpha_{1}, \alpha_{2}}^{\mathrm{ret}}\left(t_{1}, t_{2}\right)-\sum_{\beta} \tilde{U}_{\alpha_{1}, \beta} \delta G_{\beta, \alpha_{2}}^{\mathrm{ret}}\left(t_{1}, t_{2}\right)-\sum_{\beta} \int \frac{\mathrm{d} t}{\hbar} \tilde{\Sigma}_{\alpha_{1}, \beta}^{\mathrm{ret}}\left(t_{1}-t\right) \delta G_{\beta, \alpha_{2}}^{\mathrm{ret}}\left(t, t_{2}\right)= \\
\sum_{\beta} \delta U_{\alpha_{1}, \beta}\left(t_{1}\right) \tilde{G}_{\beta, \alpha_{2}}^{\mathrm{ret}}\left(t_{1}-t_{2}\right)+\sum_{\beta} \int \frac{\mathrm{d} t}{\hbar} \delta \Sigma_{\alpha_{1}, \beta}^{\mathrm{ret}}\left(t_{1}, t\right) \tilde{G}_{\beta, \alpha_{2}}^{\mathrm{ret}}\left(t-t_{2}\right)
\end{aligned}
$$

This constitutes an inhomogeneous differential equation, where the homogeneous part (left-hand side) is identical with the defining equation $\left(\mathrm{A} 3\right.$ ) for $\tilde{G}^{\text {ret }}$. Thus $\tilde{G}^{\text {ret }}$ is the corresponding Green function and the solution is given by:

$$
\delta \mathbf{G}^{\mathrm{ret}}\left(t_{1}, t_{2}\right)=\int \frac{\mathrm{d} t}{\hbar} \int \frac{\mathrm{d} t^{\prime}}{\hbar} \tilde{\mathbf{G}}^{\mathrm{ret}}\left(t_{1}-t\right)\left[\delta \mathbf{U}(t) \hbar \delta\left(t-t^{\prime}\right)+\delta \boldsymbol{\Sigma}^{\mathrm{ret}}\left(t, t^{\prime}\right)\right] \tilde{\mathbf{G}}^{\mathrm{ret}}\left(t^{\prime}-t_{2}\right)
$$

and Fourier transformation using Eq. (14) gives Eq. (15) after some algebra. $\delta G^{\text {adv }}$ is evaluated in the same way with ret replaced by adv. 
The linearization of the Keldysh relation (A2) provides us with

$$
\begin{array}{r}
\delta \mathbf{G}^{<}\left(t_{1}, t_{2}\right)=\int \frac{\mathrm{d} t}{\hbar} \int \frac{\mathrm{d} t^{\prime}}{\hbar}\left[\delta \mathbf{G}^{\mathrm{ret}}\left(t_{1}, t\right) \tilde{\boldsymbol{\Sigma}}^{<}\left(t-t^{\prime}\right) \tilde{\mathbf{G}}^{\mathrm{adv}}\left(t^{\prime}-t_{2}\right)+\tilde{\mathbf{G}}^{\mathrm{ret}}\left(t_{1}-t\right) \delta \boldsymbol{\Sigma}^{<}\left(t, t^{\prime}\right) \tilde{\mathbf{G}}^{\mathrm{adv}}\left(t^{\prime}-t_{2}\right)\right. \\
\left.+\tilde{\mathbf{G}}^{\mathrm{ret}}\left(t_{1}-t\right) \tilde{\boldsymbol{\Sigma}}^{<}\left(t-t^{\prime}\right) \delta \mathbf{G}^{\mathrm{adv}}\left(t^{\prime}, t_{2}\right)\right]
\end{array}
$$

Fourier transformation gives Eq. (16) after some algebra.

\section{APPENDIX D: CHOICE OF GAUGES}

We study the linear response to an external radiation field propagating in $y$-direction

$$
\vec{F}(\vec{r}, t)=\int \frac{\mathrm{d} \omega}{2 \pi} F(\omega) \vec{e}_{z} \mathrm{e}^{\mathrm{i} k(\omega) y-\mathrm{i} \omega t} \quad \vec{B}(\vec{r}, t)=\int \frac{\mathrm{d} \omega}{2 \pi} \frac{k(\omega)}{\omega} F(\omega) \vec{e}_{x} \mathrm{e}^{\mathrm{i} k(\omega) y-\mathrm{i} \omega t}
$$

Thus we have two reasonable choices of the electromagnetic potentials [12]:

$$
\begin{array}{lll}
\text { Coulomb gauge } & \vec{A}(\vec{r}, t)=\int \frac{\mathrm{d} \omega}{2 \pi} \frac{F(\omega)}{\mathrm{i} \omega} \vec{e}_{z} \mathrm{e}^{\mathrm{i} k(\omega) y-\mathrm{i} \omega t} & \phi(\vec{r}, t)=0 \\
\text { Lorentz gauge } & \vec{A}(\vec{r}, t)=-\int \frac{\mathrm{d} \omega}{2 \pi} \frac{k(\omega) F(\omega) z}{\omega} \vec{e}_{y} \mathrm{e}^{\mathrm{i} k(\omega) y-\mathrm{i} \omega t} & \phi(\vec{r}, t)=-F(\omega) z \mathrm{e}^{\mathrm{i} k(\omega) y-\mathrm{i} \omega t}
\end{array}
$$

In the spirit of a dipole approximation (i.e., assuming that the wavelength is large compared to the size of the active region) the terms containing $k$ are neglected, providing the expressions (17, 18) in the perturbation Hamiltonian, where quadratic terms $\propto F(\omega)^{2}$ are neglected.

[1] L. Esaki and R. Tsu, IBM J. Res. Develop. 14, 61 (1970).

[2] R. F. Kazarinov and R. A. Suris, Sov. Phys. Semicond. 5, 707 (1971).

[3] J. Faist, F. Capasso, D. L. Sivco, C. Sirtori, A. L. Hutchinson, and A. Y. Cho, Science 264, 553 (1994).

[4] R. Köhler, A. Tredicucci, F. Beltram, H. E. Beere, E. H. Linfield, A. G. Davies, D. A. Ritchie, R. C. Iotti, and F. Rossi, Nature 417, 156 (2002).

[5] C. Gmachl, F. Capasso, D. L. Sivco, and A. Y. Cho, Rep. Prog. Phys. 64, 1533 (2001).

[6] S. A. Ktitorov, G. S. Simin, and V. Y. Sindalovskii, Sov. Phys.-Sol. State 13, 1872 (1972), [Fizika Tverdogo Tela 13, 2230 (1971)].

[7] K. F. Renk, E. Schomburg, A. A. Ignatov, J. Grenzer, S. Winnerl, and K. Hofbeck, Physica B 244, 196 (1998).

[8] A. Wacker, S. J. Allen, J. S. Scott, M. C. Wanke, and A.-P. Jauho, phys. status solidi (b) 204, 95 (1997).

[9] H. Kroemer, Large-amplitude oscillation dynamics and domain suppression in a superlattice Bloch oscillator (2000), arXiv: cond-mat/0009311.

[10] S. J. Allen, J. S. Scott, M. C. Wanke, K. Maranowsky, A. C. Gossard, M. J. W. Rodwell, and D. H. Chow, in Terahertz Sources and Systems, edited by R. E. Miles, P. Harrison, and D. Lippens (Kluwer Academic Publishers, Dordrecht, 1991).

[11] A. Wacker, Phys. Rep. 357, 1 (2002).

[12] J. D. Jackson, Classical Electrodynamics (John Wiley \& Sons, New York, 1998), 3rd ed.
[13] J. R. Tucker and M. J. Feldman, Rev. Mod. Phys. 57, 1055 (1985).

[14] G. Platero and R. Aguado, Appl. Phys. Lett. 70, 3546 (1997).

[15] H. Haug and S. Koch, Quantum theory of the optical and electronic properties of semiconductors (World Scientific, Singapore, 1994).

[16] L. P. Kadanoff and G. Baym, Quantum Statistical Mechanics (Benjamin, New York, 1962).

[17] S. Schmitt-Rink, D. S. Chemla, and H. Haug, Phys. Rev. B 37, 941 (1988).

[18] H. Haug and A.-P. Jauho, Quantum Kinetics in Transport and Optics of Semiconductors (Springer, Berlin, 1996).

[19] K. Henneberger and S. W. Koch, Phys. Rev. Lett. 76, 1820 (1996).

[20] M. F. Pereira, Jr. and K. Henneberger, Phys. Rev. B 58, 2064 (1998).

[21] P. Lipavský, V. Špička, and B. Velický, Phys. Rev. B 34, 6933 (1986).

[22] S. Datta, Electronic Transport in Mesoscopic Systems (Cambridge University Press, Cambridge, 1995).

[23] R. Lake, G. Klimeck, R. C. Bowen, and D. Jovanovic, J. Appl. Phys. 81, 7845 (1997).

[24] A. Wacker and B. Y.-K. Hu, Phys. Rev. B 60, 16039 (1999).

[25] C. Sirtori, P. Kruck, S. Barbieri, P. Collot, J. Nagle, M. Beck, J. Faist, and U. Oesterle, Appl. Phys. Lett. 
73, 3486 (1998).

[26] A. Wacker, A.-P. Jauho, S. Rott, A. Markus, P. Binder, and G. H. Döhler, Phys. Rev. Lett. 83, 836 (1999).

[27] S.-C. Lee and A. Wacker, Physica E 13, 858 (2002).

[28] L. L. Bonilla, in Nonlinear Dynamics and Pattern Formation in Semiconductors and Devices, edited by F.-J. Niedernostheide (Springer, Berlin, 1995), chap. 1, pp. 120.

[29] M. Patra, G. Schwarz, and E. Schöll, Phys. Rev. B 57, 1824 (1998).

[30] R. C. Iotti and F. Rossi, Phys. Rev. Lett. 87, 146603
(2001)

[31] A. Y. Shik, Sov. Phys. Semicond. 8, 1195 (1975), [Fiz. Tekh. Poluprov. 8, 1841 (1974)].

[32] A. Wacker and A.-P. Jauho, Phys. Rev. Lett. 80, 369 (1998).

[33] F. Eickemeyer, R. A. Kaindl, M. Woerner, T. Elsaesser, S. Barbieri, P. Kruck, C. Sirtori, and J. Nagle, Appl. Phys. Lett. 76, 3254 (2000).

[34] Note that the translational symmetry of the structure is broken in the Lorentz gauge 